\title{
A PUBLIC PLATFORM FOR GEOSPATIAL DATA SHARING FOR DISASTER RISK MANAGEMENT
}

\author{
S. Balbo ${ }^{a^{*}}$, P. Boccardo ${ }^{b}$, S. Dalmasso ${ }^{a}$, P. Pasquali ${ }^{\text {a }}$ \\ a ITHACA, via Pier Carlo Boggio 61, 10138 Torino, Italy - (simone.balbo, simone.dalmasso, \\ paolo.pasquali)@ithaca.polito.it \\ ${ }^{\mathrm{b}}$ Interuniversity Department of Regional and Urban Studies and Planning, Politecnico di Torino, Corso Duca degli \\ Abruzzi 24, 10129 Torino, Italy - piero.boccardo@polito.it
}

KEY WORDS: GeoNode, Geospatial Data Sharing, Spatial Data Infrastructure, Web GIS

\begin{abstract}
:
Several studies have been conducted in Africa to assist local governments in addressing the risk situation related to natural hazards. Geospatial data containing information on vulnerability, impacts, climate change, disaster risk reduction is usually part of the output of such studies and is valuable to national and international organizations to reduce the risks and mitigate the impacts of disasters. Nevertheless this data isn't efficiently widely distributed and often resides in remote storage solutions hardly reachable.

Spatial Data Infrastructures are technical solutions capable to solve this issue, by storing geospatial data and making them widely available through the internet. Among these solutions, GeoNode, an open source online platform for geospatial data sharing, has been developed in recent years. GeoNode is a platform for the management and publication of geospatial data. It brings together mature and stable open-source software projects under a consistent and easy-to-use interface allowing users, with little training, to quickly and easily share data and create interactive maps. GeoNode data management tools allow for integrated creation of data, metadata, and map visualizations. Each dataset in the system can be shared publicly or restricted to allow access to only specific users. Social features like user profiles and commenting and rating systems allow for the development of communities around each platform to facilitate the use, management, and quality control of the data the GeoNode instance contains (geonode.org).

This paper presents a case study scenario of setting up a Web platform based on GeoNode. It is a public platform called MASDAP and promoted by the Government of Malawi in order to support development of the country and build resilience against natural disasters. A substantial amount of geospatial data has already been collected about hydrogeological risk, as well as several otherdisasters related information. Moreover this platform will help to ensure that the data created by a number of past or ongoing projects is maintained and that this information remains accessible and useful. An Integrated Flood Risk Management Plan for a river basin has already been included in the platform and other data from future disaster risk management projects will be added as well.
\end{abstract}

\section{DISASTER RISK IN MALAWI AND THE SHIRE RIVER BASIN}

Malawi has been traditionally vulnerable to natural disasters because of its unique geoclimatic conditions. In view of the country's high vulnerability profile, the recurrent phenomena in the form of hydro-meteorological hazards affects millions across the country leaving behind a trail of losses in the form of lives, property and livelihoods. In part of the country, disaster losses tend to outweigh the development gains. The economic and social losses because of droughts and floods continue to mount year after year (RMSI, 2009).

Malawi is affected by a number of natural and human-made disasters every year and between 1974 and 2003, hazards cumulatively affected 25 million people (Atkins, 2011). This vulnerability is a result of a combination of physical geoclimatic factors, environmental factors and socio-economic vulnerability. There is widespread dependence on rain-fed agriculture and use of biomass for household energy, and the nation has a narrow economic base and extensive rural poverty. The intensity, frequency and number of people affected by such disasters appears to be increasing, a trend attributed to climate change, population growth, urbanisation and environmental degradation (Atkins, 2011).
A number of efforts are being implemented by Malawi government and other stakeholders to reduce the risks and mitigate the impacts of disasters. In 2009 RMSI, funded by the Global Facility for Disaster Recovery and Reduction (GFDRR, see Section 2) of the World Bank, produced a report on drought and flood vulnerability. They calculated the losses in maize and tobacco based on the results from stochastic flood models. For the flood vulnerability assessment, they focused their flood modelling on the Shire River basin.

The Shire River, which is the outlet of Lake Malawi and flows into the Zambezi, and its tributaries are a major source of flooding in the country, with the highest flooding frequency occurring in the Lower Shire valley. The Shire Valley i.e. southern Malawi is regularly ravaged by floods that adversely affect local populations, mostly poor subsistence farmers, by causing loss of lives and destroying property including settlements, social, road, rail and other national infrastructure. Out of a total population of 13 million of Malawi, the southern region has the highest population of 5.8 million (45\%). It has also the highest number of households ( 1.4 million) from a total of 3 million in the country. Flooding has resulted in higher poverty and vulnerability levels in high risk districts (RMSI, 2009).

\footnotetext{
* Corresponding author
} 
Since the 1970s, flooding in the basin has occurred once in every 2 - 5 years. The Shire River is economically and environmentally very important, with hydroelectric schemes generating $98 \%$ of Malawi's electricity, extensive fisheries and wildlife conservation areas and provision of freshwater irrigation to cash crop plantations, industrial and domestic uses, and is therefore of national importance (Atkins, 2011).

If vulnerabilities and hazards could be addressed, intensified agricultural production, better transport linkages and secured homesteads can make the valley an economic hub of Malawi and the region, and lift many people out of poverty. In the short and medium term the focus should be on building interventions on a better understanding of the risk profiles, and mitigating immediate loss that occur too frequently.

In order to harmonize efforts that are being implemented in isolation by different agencies in the Shire basin, yielding little visible impact overall, and to improve the community participation in catchment protection and management, the government together with the World Bank initiated the Shire River Basin Management Program (SRBMP).

The overall objective of the Program is to increase sustainable social, economic and environmental benefits by effectively and collaboratively planning, developing and managing the Shire River Basin's natural resources. It will have a duration of 15 years. The first phase project will establish coordinated intersectorial development planning and coordination mechanisms, undertake the most urgent water related infrastructure investments, prepare additional infrastructure investments, and develop up-scalable systems and methods to rehabilitate subcatchments and protect existing natural forests, wetlands and biodiversity, thereby beginning to address some of the most critical issues facing the Basin (World Bank, 2012).

One of the most relevant project objectives is to improve flood management in the Lower Shire and provide community level adaptation and mitigation support. One of the first results of the project is the Integrated Flood Risk Management Plan for the Shire basin that the World Bank commissioned Atkins to develop with other governmental departments. It will result in an action plan to address the flood risk situation in the Lower Shire in an integrated way to assess and implement future interventions. The plan should address both the hazard in terms of water regime and the vulnerability to these hazards as adaptation of human behaviour.

As a result of this project and of the RMSI's, a remarkable amount of geospatial data has been collated and produced, both in terms of base reference data, such as hydrology, infrastructures etc., and in terms of thematic data, such as probabilistic flood extent area and flood risk classification of the territory.

\section{OPENDRI AND THE GEONODE APPLICATION}

The Global Facility for Disaster Recovery and Reduction (GFDRR), a partnership of 41 countries and 8 international organizations managed by the World Bank and committed to helping developing countries reduce their vulnerability to natural hazards and adapt to climate change, leads the Open Data for Resilience Initiative (OpenDRI; GFDRR, 2010). The OpenDRI aims to reduce the impact of disasters by empowering decisions-makers with better information and the tools to support their decisions (GFDRR, 2013).

Indeed in order to build disaster resilient societies, policymakers and the public must have access to the right data and information to inform good decisions. Examples of such decisions include where and how to build safer schools, how to insure farmers against drought, and how to protect coastal cities against future climate impacts. Sharing data and creating open systems promotes transparency, accountability, and ensures a wide range of actors are able to participate in the challenge of building resilience (GFDRR, 2013).

The OpenDRI initiative takes advantage of the GeoNode (GeoNode, 2013) as the operational platform that supports its implementation; it is currently being adopted in many developing countries at risk from natural disasters, such as Haiti, Kenya, Saint Vincent and the Grenadines, Grenada, Trinidad and Tobago, for data sharing among various agencies, scientific and governmental institutions at a country level. This action has two advantages: on the one hand duplication of efforts is avoided in data collection, validation and management, while on the other hand high reliability is expected from the data since the responsibility of data related to specific areas of information is assigned to experts in the respective sector.

In previous section it has been said that in Malawi a variety of studies have been conducted to better understand the vulnerability to natural hazards and the effects of climate change, as well as the status of its natural resources on which the majority of rural livelihoods and the economy rely, through several World Bank and other international efforts in recent years. As part of these studies, important datasets related to vulnerability, impacts, climate change, disaster risk reduction and preparedness have been collected and created. All too often this data has not been widely distributed or made available to the Governmental departments or other important actors involved in building resilience in the country (World Bank, 2011).

In the framework of the SRBMP the World Bank is partnering with the Government of Malawi to carry on a project that will help contribute to the reduction of flood risk in the Shire River Basin. A working group involving a number of key ministries has been active in the design of the project and it is anticipated that a significant amount of new spatial data will be created over the course of the project (World Bank, 2011).

In partnership with the World Bank's OpenDRI and with the technical collaboration of ITHACA, a non-profit association that works on acquisition, management and elaboration of geographic and cartographic data (ITHACA, 2013), the governmental departments have decided in 2012 to launch an online platform, called MASDAP, to help ensure that the data created by a number of past or ongoing projects is maintained so that this information remains accessible and useful to the Government. This platform is based on the free and open-source software, GeoNode.

\section{MASDAP: DESCRIPTION AND FUNCTIONALITIES}

The Malawi Spatial Data Platform (MASDAP) is a userfriendly platform based on free and open-source software that will enable improved inter-agency information collaboration and enhanced public access for awareness-building, research, further knowledge product creation and decision support. The access is publicly available on the internet: www.masdap.mw. A screenshot of the homepage can be seen in Figure 1.

It is based on an ad-hoc look and feel customization of the GeoNode application as well as a re-organization of the main functionalities access points. Its main functionalities, a standard for the GeoNode application, are: geospatial data search, geospatial data exploration, metadata management, map creation, map exploration, users and profiles management. 
The GeoNode provides the user with a series of functionalities in the field of geomatics that are typical of a desktop GIS application, such as creating and printing maps. The capacity of accessing functionalities, traditionally provided by desktop applications, from the Web is a technical solution called "Software as a Service", that is expected to increase in the next future and to overcome the common strategy of desktop applications (Patrignani N., 2009). The aim is to simplify the management of desktop computers, moving workload towards computer servers, and to promote data sharing among users since with this approach data are centralized and can be easily accessed from many computer clients even in concurrent mode.

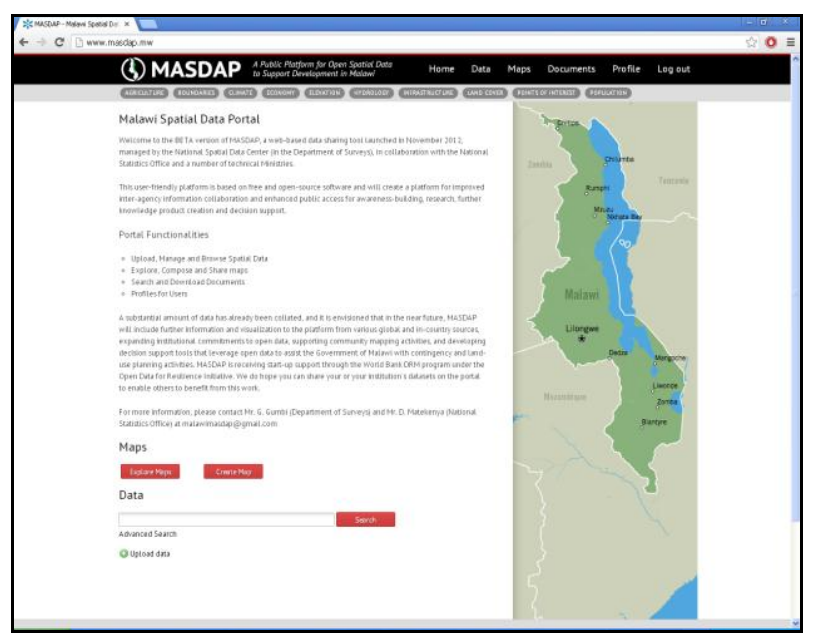

Figure 1. Screenshot of the MASDAP platform homepage

\subsection{Data sharing}

The promotion of data sharing is among the first objectives of the community of GeoNode developers. On the one hand data sharing means that a user is put in condition of uploading data into the system and to give other users the possibility of exploring these data. On the other hand the user is provided with the capacity of taking advantage of data shared by others either through online tools or by downloading them locally in formats suitable for desktop applications (Pirotti et al., 2011).

3.1.1 Data uploading: data can be uploaded by registered users through a simple web interface; with the last official release, GeoNode permits to upload geo-spatial data in the following formats:

- geoTiff, it is a file format for storing georeferenced raster data;

- shapefile, it is the Esri format for georeferenced vector data, a standard de facto among the GIS community.

Moreover for each data the Web interface permits also to upload the style to be used during graphical rendering of the data themselves, encoded into an open format which is called SLD that is Styled Layer Descriptor. Furthermore during the upload process data are converted and then stored into the server in formats suitable for efficient storing and future retrieving: this step is transparent to the user.
3.1.2 Data downloading: data can be found on the platform by exploiting the embedded search engine, which allows combining classical search on a keyword basis with spatial search, by graphically defining a bounding box on the computer screen. Once interesting data have been found, the user is prompted to the so called data page: it is a Web page where data are graphically rendered in a frame, over a base layer that is retrieved dynamically from available free Web services, for example Google Maps, Open Street Map and NASA Blue Marble. This frame contains also tools for zooming and panning the spatial content as well as a querying tool for data semantic content.

The graphical rendering is devolved upon GeoServer (see Section 4 for details), that is a Web mapping server. The user can take advantage of this technology and download data by simply clicking on the specific link on the right-hand side of the data page. With the last release of the GeoNode raster data can be downloaded as geoTiff, JPEG, PDF, PNG, KML. Instead vector data can be downloaded as shapefile, GML, CSW, Excel, GeoJSON, JPEG, PDF, PNG, KML.

Other interesting elements displayed on the data page are the metadata, that are showed partially on top and partially below the frame, and the style manager application that allows to change the way data are displayed. Furthermore, according to the permission level of the current user, several links for data management and permission assignment are displayed on the right-hand side. A screenshot of the mentioned data page is showed in Figure 2.

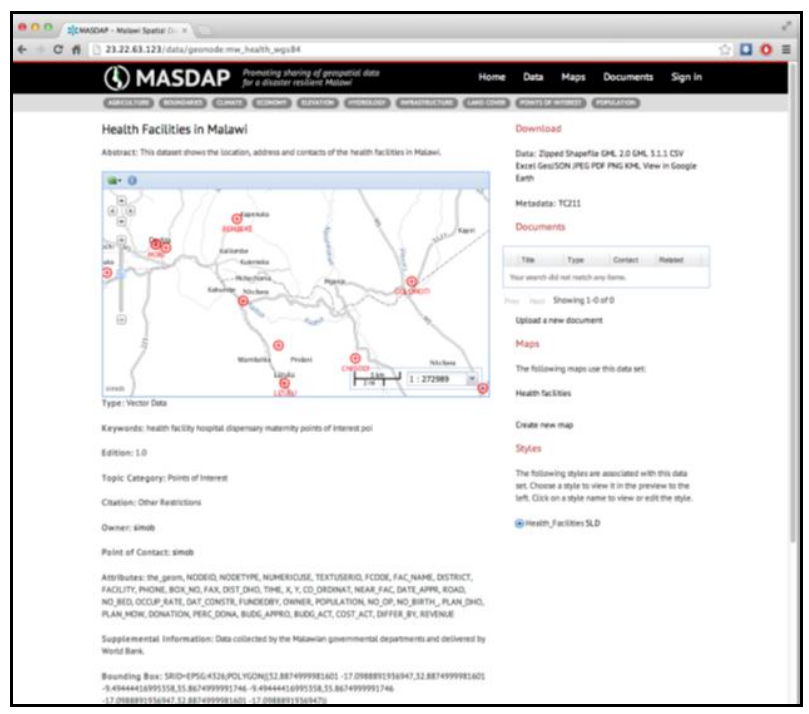

Figure 2. Screenshot of the MASDAP page for Health Facilities data.

\subsection{Creating maps}

Registered users can create Web maps by collecting together a number of data of interest and styling them in the favourite way. Maps in GeoNode are ordered collection of layers with a defined style, which are rendered to a protocol suitable for visualization in a Web browser. The Web interface for map creation makes this operation similar to the most common desktop GIS applications (see Figure 3). Once a map is created it can be saved and stored into the platform, in order to be easily retrieved, explored and potentially modified in the future. Maps can also be easily published on blogs and other websites and they can be printed to a pdf format.

Moreover maps existent on the platform can be searched by means of a search engine based on keywords; when a map of 
interest is found, the user is prompted to the so called map page. This is a Web page that shows a preview of the map itself in a frame on the left-hand side of the screen; on top of it the map title and an abstract are displayed. Furthermore the list of layers contained in the map as well as the link for data downloading are displayed on the right-hand side of the screen. Finally, similarly to what happens with data, special commands for map management and permission assignment are showed.

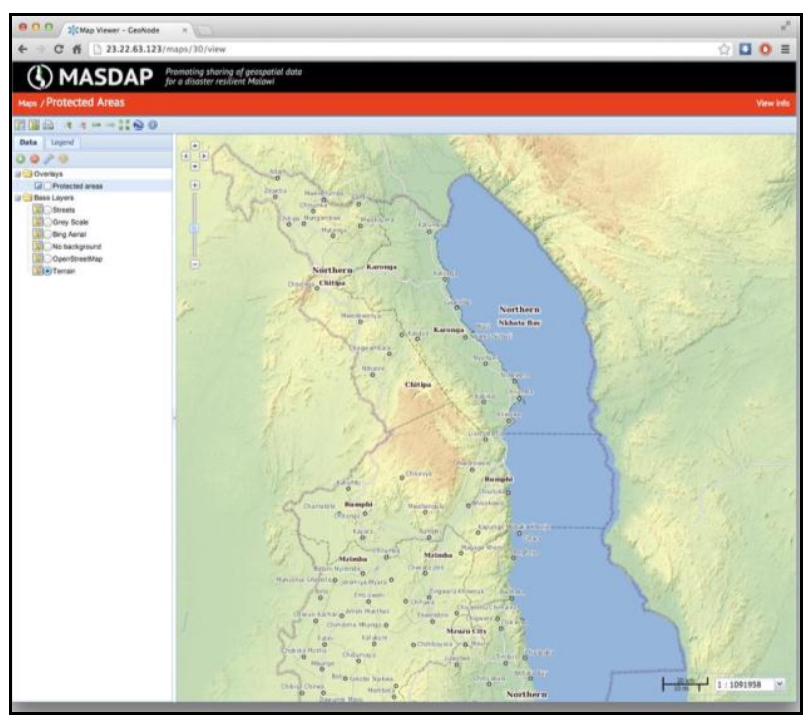

Figure 3. Screenshot of the map composer application of the MASDAP platform

\subsection{Metadata management}

During data uploading, the user is prompted to fill in a metadata form that will be showed to the users who will look for those data in the future. The same form can be used every time it may be needed for updating the metadata content.

The latest GeoNode release can manage a metadata form which contains a subset of the collection of fields suggested in the ISO standards for geo-spatial data (see Section 4 for details). Nevertheless this form can be edited and developed in order to match other standard or user-specific formats.

\subsection{Data sensitivity}

Data sensitivity is a key issue in every environment that involves data exchange and publication. It has to be guaranteed especially in case of spatial data related to emergency management.

The GeoNode implements a framework of users, groups and permissions typical of a DBMS; this is due to the Web framework that ensures fast, direct and reliable reading and writing to a DBMS which runs in support of the Web platform. Every user can access and potentially edit only data which he's allowed to see and manage. On the other hand the user who uploads data become its owner on the platform and can assign permissions to other users and groups. The system administrator has the possibility of making changes to the permissions in case of need.

\section{GEONODE-MASDAP ARCHITECTURE}

The GeoNode platform as a whole builds upon several open source software products already developed and combines them together in order to take advantage of their functionalities in an integrated manner.

In particular the GeoNode is made of the following components:

- A Database Management System and its spatial data extension: PostgreSQL and PostGIS;

- A server-side software which can provide standard Web Map Services: GeoServer;

- $\quad$ A metadata catalogue software: GeoNetwork;

- A Web-platform framework: Django;

- Client-side libraries for building WebGIS applications: OpenLayers and GeoExt.

In the following sections these components are introduced and their role in the platform is described. A schema of the software architecture is contained in Figure 4.

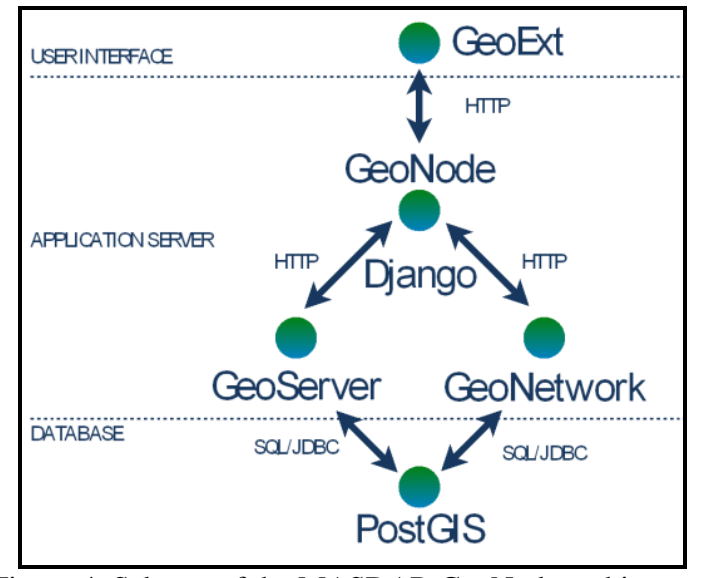

Figure 4. Schema of the MASDAP-GeoNode architecture

\subsection{PostgreSQL and PostGIS}

PostgreSQL is a free and open source object-relational database management system available for many computer platforms. It is developed by a global development group, consisting of a handful of community volunteers employed and supervised by large companies in the software development field. It implements the majority of the SQL:2008 standard, is fully transactional, includes all Data Definition Language statements, has extensible data types, operators and indexes (PostgreSQL, 2013).

PostGIS is an extension to PostgreSQL, developed in the framework of a spatial database technology research project, which allows GIS objects to be stored into the database. In effect, PostGIS "spatially enables" the PostgreSQL server, allowing it to be used as a backend spatial database for geographic information systems. It includes support for GiSTbased R-Tree spatial indexes and functions for analysis and processing of GIS objects. Last development have added user interface tools, basic topology support, data validation, coordinate transformation and programming among other functionalities (PostGIS, 2013).

In GeoNode PostgreSQL is used with a double function: on the one hand it works in support of the GeoNode website, being the place where all the information about users and groups is stored and the instrument that assure the compliance to the defined user permissions. Moreover it contains also all the information that regards specifically the dynamic content of the website such as sentence translations in various languages. On the other hand it works as data repository for geospatial data by means of the PostGIS extension, in particular for vector data. Relevant advantages can be detected when storing vector data in 
PostGIS, rather than storing them in filesystem, both in terms of performances in data selection and management. On the raster data side instead, even if the last PostGIS version allows raster storage, they are simply stored in filesystem.

\subsection{GeoServer}

GeoServer is an open source software server written in Java that allows users to publish geospatial data. It is designed for interoperability and publishes data from any major spatial data source using open standards. Being a community-driven project, GeoServer is developed, tested, and supported by a diverse group of individuals and organizations from around the world. Furthermore it is the reference implementation of the Open Geospatial Consortium Web Feature Service (WFS) and Web Coverage Service (WCS) standards, as well as a high performance certified compliant Web Map Service (WMS) (GeoServer, 2013).

GeoServer is the map engine in support of the GeoNode, it works on the server side of the system and performs three main activities:

1. it renders all the geospatial content showed by the GeoNode in a graphic format, in particular in the data page, in the map page and in the map composer;

2. it performs all the data format transformations when it comes to data downloading in formats that are different from the original source;

3. it enables the GeoNode as a WFS and WMS server, therefore data can be served with standard Web services by the platform.

\subsection{GeoNetwork Opensource}

GeoNetwork Opensource is a standard based and decentralised spatial information management system, designed to enable access to geo-referenced databases and cartographic products from a variety of data providers through descriptive metadata, enhancing the spatial information exchange and sharing between organisations and their audience, using the capacities and the power of the Internet. Its main features are:

- instant search on local and distributed geospatial catalogues;

- online metadata editing with a powerful template system;

- scheduled metadata harvesting and synchronisation between distributed catalogues;

- groups and users management;

- fine grained access control.

It is compliant with the metadata standard ISO19115:2003, using the ISO19139:2007 implementation specification schema published in May 2007. The release also serves as the open source reference implementation of the OGC Catalogue Service for the Web (CSW 2.0.2) (GeoNetwork, 2013).

In particular the GeoNode takes advantage of two main functionalities that are implemented in GeoNetwork: the two search engines. The first is a typical Web search engine and works on the basis of some keywords that are assigned to each dataset and then retrieved when submitted in the search string. The second is a spatial search engine by means of which it is possible to search for data covering a certain area of interest, on the basis of its geographic extent.

\subsection{Django}

It has been said that GeoNode combines together many software products which are already existing and sufficiently stable to be used by a wide community of users. Being a Web platform, the main GeoNode component is the code that describes the website logic. This code is written in Python programming language and takes advantage of a framework for Web development called Django.

Django encourages rapid development and clean, pragmatic design and applies the DRY principle. Duplication, both inadvertent and purposeful, can lead to maintenance difficulties, poor factoring and logical contradictions. It can arise in architecture, requirements, code or documentation, with effects that can range from mis-implemented code and developer confusion to complete system failure. The DRY principle, that is an acronym of Don't Repeat Yourself states that "every piece of knowledge must have a single, unambiguous, authoritative representation within a system" (c2 wiki, 2011).

Moreover Django adheres to the Model-View-Controller software architecture, an architectural pattern used in software engineering that isolates the application logic for the user from the user interface, permitting independent development, testing and maintenance of each part (Django, 2013).

\subsection{GeoExt and OpenLayers}

GeoExt is an open source JavaScript toolkit aimed at building rich Web mapping applications. It brings together the geospatial know how of OpenLayers with the user interface savvy of ExtJS to help build powerful desktop style GIS applications on the Web with JavaScript (GeoExt, 2013).

On the one hand GeoExt extends Ext JS, a rich library of web UI widgets and helper classes: in details it is a JavaScript framework for building rich application in Web browsers, that enables developers to use a Model-View-Controller architecture when building their application, and offers a wide range of user interface widgets such as scalable grids, trees and menus (Sencha, 2013).

On the other hand the OpenLayers mapping library is the key component of GeoExt, performing the core map-related functions of every GeoExt-based application.

OpenLayers is a pure JavaScript library for displaying map data in most modern Web browsers; it hasn't any server-side dependencies, and is developed and supported by a number of organizations around the world (OpenLayers, 2013).

OpenLayers and GeoExt are used in GeoNode on the client side, in particular in the Web pages that show cartographic representation of data and maps; therefore they are used in the GeoNode data and map page and in the map composer application. Examples of OpenLayers functions are the zoom and map options in the map visualization.

\section{CONCLUSIONS}

This article introduced the Malawi Spatial Data Platform, a public Web platform aimed at sharing and publishing geospatial data in the field of disaster risk management.

In Section 1 the flood risk situation in Malawi and in particular in the Shire River Basin has been introduced. The key point is the vulnerability of the country to natural disasters such as droughts and especially floods, and consequently the intervention of the Government and other actors in terms of financing several studies and investing in environmental management programs in order to build resilience against floods in the Shire River Basin. As a result of these studies a quantity of geospatial data were and are going to be produced.

In Section 2 the OpenDRI initiative and the needs in the country of Malawi in terms of opening and sharing data related 
to floods and other disasters have been introduced. The key point is the need to effectively share and make public and actually useful to everybody the data coming from the studies previously mentioned. This is strongly useful in the response step of the disaster cycle but also in the other steps, such as preparedness and recovery.

In Section 3 the dissertation focused on the MASDAP platform and on its functionalities. The key point is the operational solution that has been implemented by the Government and by the World Bank office in order to make the geospatial data public in an effective way; a public Web platform for geospatial data publishing and sharing was set up and is now operational, after its official launch in 2012. It allows to investigate the content in terms of data and maps and to download the content itself in order to take their maximum advantage; moreover it allows to actively participate to the platform by uploading and creating additional content to be shared with others. The final aim of the platform is to be used as an everyday instrument for governmental departments and other institutional actors for sharing geospatial data and related content.

Finally, in Section 4, the MASDAP platform has been described from the architectural point of view, detailing the software components and their role in the whole application. In particular MASDAP is the result of the look and feel customization, the re-organization of the functionalities access points, and the Malawi-specific data-content filling of the standard GeoNode application.

Four conclusive elements can be introduced: firstly the technical solution described throughout this article is capable to solve the need of opening geospatial data to the public in the country of Malawi. Several datasets are currently available on the platform and the main functionalities have been extensively tested. Nevertheless the project is at an early stage therefore no users' feedback on production performance is available yet.

Secondly the following activities are going to focus on flood preparedness by collecting and sharing data from ad hoc surveys on the vulnerable assets such as residential buildings, key infrastructures and production sites. Moreover the use of the platform and its advantages in a flood response phase has to be tested yet.

Thirdly two main critical aspects should be highlighted: on the one hand the internet connectivity is a key issue since the MASDAP platform is an online tool, therefore it is effective only when a minimum threshold bandwidth is guaranteed. Nevertheless the general trend is to have faster internet connectivity in the near future. On the other hand is the scepticism of several governmental departments towards opening data. Even if the platform con manage data sensitivity through a DBMS like permission approach, its use is sometimes perceived as an unsafe way of data distribution and eventually a potential economic loss because some data have been on sell so far even among governmental departments.

Fourthly the MASDAP platform brings an indirect advantage that is the effort spent in capacity building in the country, especially in the field of geomatics. Indeed a MASDAP technical team was created by putting together several representatives of the governmental departments involved in GIS and remote sensing; moreover two persons from this group were assigned the role of platform administrators. Also two training sessions were held to the technical team on the use of the platform and more generally on several topics in the field of GIS, IT and Web sites. This effort is likely to involve the administrators into international communities of open source software in the field of geomatics and to incite the technical team in data sharing for disaster management.

\section{REFERENCES}

Atkins, 2011. Integrated Flood Risk Management Plan for the Shire Basin project. Inception Report - Final. Atkins Limited, 500 Park Avenue, Almondsbury, Bristol, BS32 - 4RZ, England, pp 1-19.

Balbo, S., 2012. A reference data access service in support of emergency management - Data quality assessment protocol, publication and exploitation of the results. $\mathrm{PhD}$ Final Dissertation. Politecnico di Torino. Chapter 7.

GFDRR, 2010. Partnership Charter. Global Facility for Disaster Reduction and Recovery. Washington, DC. USA.

Patrignani N., 2009. Computer Ethics. PhD course 2009, Politecnico di Torino. Course presentations.

Pirotti, F., Guarnieri, A., Vettore, A., 2011. Collaborative WebGIS design: a case study for road risk analysis and monitoring. Transactions in GIS 15(2), pp. 213-226.

RMSI, 2009. Malawi: Economic Vulnerability and Disaster Risk Assessment. Final Report. RMSI, Noida 201301, INDIA. Pp. 21-28

World Bank, 2011. Malawi Disaster and Natural Resources Spatial Data Sharing Platform - Terms of Reference.

World Bank, 2012. Malawi - Adaptable Program Loan for Shire River Basin Management Program Project. Project Appraisal Document. Pp. 1-14

\section{References from websites:}

c2 wiki, 2011. Don't Repeat Yourself. Cunningham \& Cunningham Inc. http://c2.com/cgi/wiki?DontRepeatYourself (22 Nov. 2011)

Django, 2013. Django Documentation, Version 1.4. https://docs.djangoproject.com/en/1.4/ (8 Feb. 2013)

GeoExt, 2013. GeoExt - Documentation. Core Concepts. http://geoext.org/primers/index.html (3 February, 2012)

GeoNetwork, 2013. GeoNetwork Opensource, User and Developer Manuals, Version 2.6.4. http://geonetworkopensource.org/docs.html (8 Feb. 2013)

GeoNode, 2013. GeoNode gallery page. http://geonode.org/gallery/ (8 Feb. 2013)

GeoServer, 2013. What is GeoServer. http://geoserver.org/display/GEOS/What+is+GeoServer ( 8 Feb. 2013)

GFDRR, 2013. Open Data for Resilience Initiative. https://www.gfdrr.org/opendri (6 Feb. 2013)

ITHACA, 2013. About. http://www.ithacaweb.org/about/ (8 Feb. 2013)

OpenLayers, 2013. OpenLayers homepage. http://openlayers.org/ ( 30 Jan. 2012)

PostGIS, 2013. PostGIS 1.5 Manual. http://postgis.net/docs/manual-1.5/ (8 Feb. 2013) 
The International Archives of the Photogrammetry, Remote Sensing and Spatial Information Sciences, Volume XL-5/W3, 2013 The Role of Geomatics in Hydrogeological Risk, 27 - 28 February 2013, Padua, Italy

PostgreSQL, 2013. PostgreSQL 9.1 comprehensive manual. http://www.postgresql.org/docs/9.1/static/index.html (8 Feb. 2013)

Sencha, 2013. Sencha - Products Overview: ExtJS4. http://www.sencha.com/products/extjs/ (3 Feb. 2012)

\section{ACKNOWLEDGEMENTS}

The author would like to thank the Government of Malawi and the local World Bank offices for the significant amount of information provided. 